\title{
Comprimento do colo uterino e dilatação do orificio interno avaliados pela ultra-sonografia bidimensional e tridimensional
}

\author{
Cervical length and internal cervical os dilatation evaluated \\ by two-dimensional and three-dimensional ultrasound
}

\begin{abstract}
Rosieny Souza Brandão ${ }^{1}$, Carlos Geraldo Viana Murta ${ }^{2}$, Antonio Fernandes Moron ${ }^{3}$, Rosiane Mattar ${ }^{4}$, Claudio Rodrigues Pires ${ }^{5}$, Eduardo Almeida Guerzet ${ }^{6}$
\end{abstract}

\section{RESUMO}

Objetivo: comparar as medidas do comprimento da cérvice e afunilamento, obtidas por ultra-sonografia transvaginal bidimensional e tridimensional na gestação. Métodos: estudo descritivo, prospectivo, com comparação de grupos realizado no período de abril de 2004 a fevereiro de 2005. Foram incluídas 74 gestantes com idade gestacional entre a $19^{\mathrm{a}}$ e a $24^{a}$ semana, independente da presença ou não de fatores de risco para parto prematuro. O exame ultra-sonográfico da cérvice foi efetuado uma única vez na mesma paciente e por único observador. As medidas aferidas por ultra-sonografia bidimensional foram feitas no momento da realização dos exames, e pela tridimensional, com intervalo de 7 a 15 dias do exame bidimensional. A medição do comprimento da cérvice, diâmetro e comprimento do funil cervical foi realizada por ultra-sonografia bidimensional no plano sagital e tridimensional nos planos sagital e coronal. Para testar a diferença estatística entre os resultados médios das medidas do comprimento cervical obtidas nos exames bi e tridimensional, foi utilizado o teste $t$ pareado. Também foi calculado para essas medidas o coeficiente de correlação de Pearson. Resultados: objetivando-se determinar associação linear entre elas encontramos diferença significante entre as medidas aferidas no plano sagital por meio da ultra-sonografia bidimensional e plano coronal da USG 3D ( $p=0,009)$, e na ultra-sonografia tridimensional entre os cortes sagital e coronal $(\mathrm{p}=0,001)$, sendo as médias das medidas do comprimento cervical ( \pm desvio

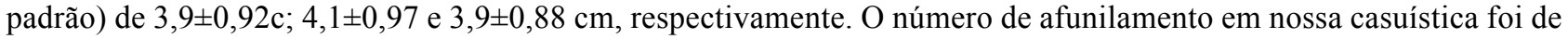
$9 / 74$ exames $(12,1 \%)$. As ultra-sonogafias bi- e tridimensional visualizaram o mesmo número de afunilamento (8/9). Na ultra-sonografia tridimensional, o corte coronal detectou número maior de funil cervical que o corte sagital (8/9). Não houve diferença significativa entre as médias das medidas do comprimento do colo aferidas por ultra-sonografia bidimensional e tridimensional no plano sagital $(\mathrm{p}=0,23)$, entretanto houve diferença entre as médias das medidas da cérvice mensuradas por ultra-sonografia bidimensional no corte sagital e tridimensional no coronal $(\mathrm{p}=0,009)$, e na ultra-sonografia tridimensional entre os planos sagital e coronal $(\mathrm{p}=0,001)$. Os exames bidimensional e tridimensional visualizaram o mesmo número de funil cervical, demonstrando concordância entre os métodos (teste kappa=0,86). Na comparação das médias das medidas do afunilamento, não houve diferença estatística $(p>0,05)$. Conclusão: existem diferenças entre as medidas do comprimento cervical, obtidas por ultra-sonografia bidimensional e tridimensional, testando o plano coronal do exame tridimensional.

PALAVRAS-CHAVE: Colo do útero/ultrasonografia; Gravidez; Ultra-sonografia

\section{ABSTRACT}

Purpose: to compare the uterine cervix measurements and funneling obtained by two- and three-dimensional transvaginal sonography during pregnancy. Methods: a prospective, descriptive study, with group comparison, was carried out between April 2004 and February 2005 in 74 pregnant women, who were between the 19th and 24th week of pregnancy,

Universidade Federal de São Paulo - Escola Paulista de Medicina - UNIFESP - São Paulo (SP), Brasil.


- Vitória (ES), Brasil.

2 Professor do Departamento de Ginecologia e Obstetrícia da Universidade Federal do Espírito Santo - UFES - Vitória (ES), Brasil.

3 Professor Titular do Departamento de Obstetrícia da Universidade Federal de São Paulo - UNIFESP - São Paulo (SP), Brasil.

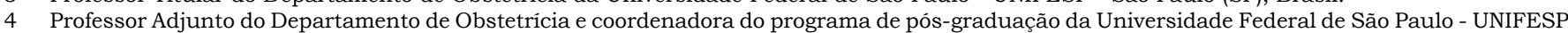
- São Paulo (SP), Brasil.

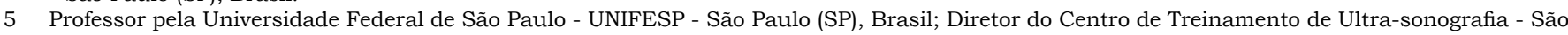
Paulo (SP), Brasil.

6 Professor Adjunto do Departamento de Tocoginecologia da Escola de Medicina da Santa Casa de Misericórdia de Vitória - EMESCAM - Vitória (ES), Brasil. Correspondência: Rosieny Souza Brandão

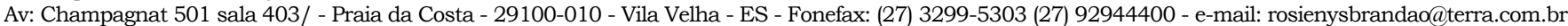


regardless of risk factors for premature delivery. The ultrasound examination of the cervix was carried out only once in the same patient and by only one observer. The measurements of the cervix by two-dimensional ultrasound were made at the time of the test and by three-dimensional ultrasound at intervals of 7 to 15 days after the two-dimensional ultrasound. The measurements of cervical length, funnel width and length were taken by two-dimensional ultrasound in the sagittal plane and by three-dimensional in the sagittal plane as well as in coronal plane. Results: there was no significant difference between the averages of the measurements of the cervix obtained by two- and three-dimensional ultrasound in the sagittal plane ( $\mathrm{p}=0.23)$; however, there was a difference in the averages of the measurements of the cervix obtained by two-dimensional ultrasound in sagittal plane and three-dimensional ultrasound in the coronal plane $(p=0.009)$ and between three-dimensional ultrasound in the sagittal and coronal planes $(\mathrm{p}=0.001)$. The kappa test $(0.86)$ showed no superiority of either the two-dimensional and three-dimensional ultrasound in the visualization of the cervical funnel. No statistically significant difference was observed between the methods when the average of the measurements of funneling was compared ( $p>0.05$ ). Conclusion: there were differences between two-dimensional and three-dimensional ultrasound of cervical length, only using the coronal plane of the three-dimensional ultrasound.

KEYWORDS: Cervix uteri/ultrasonography; Pregnancy; Ultrasonography

\section{Introdução}

Desde a década de 70 , a ultra-sonografia bidimensional (USG 2D) foi utilizada no estudo do colo uterino durante a gestação, para identificar as modificações estruturais da cérvice no transcorrer da gravide $z^{1,2}$.A princípio, os exames eram realizados pela via abdominal ${ }^{1}$ e perineal ${ }^{3}$. Entretanto, no final dos anos 80, a evolução dos transdutores e o advento da sonda ultra-sonográfica endovaginal de alta freqüência e utilizada próxima ao colo possibilitaram avaliação cervical detalhada de forma mais precisa do que pela via abdominal ${ }^{4,5}$.

O comprimento do colo uterino mantém-se inalterado até o final do segundo trimestre, diminuindo, a partir desde momento, lenta e progressivamente até o termo ${ }^{6}$. Em decorrência disso, a detecção precoce de encurtamento da cérvice é considerado marcador de risco de parto prematuro ${ }^{7}$, sendo o risco de parto pré-termo inversamente proporcional à medida do comprimento cervical aferido por USG (ultra-sonografia) endovaginal ${ }^{8-10}$.

No final dos anos 90 a USG tridimensional (USG 3D) foi introduzida no estudo da cérvice durante a gravide $z^{11}$. Alguns pesquisadores publicaram pela primeira vez resultados referentes à análise da biometria cervical por ecografia 2D e 3D, em gestantes, comparando ambas as técnicas de exames ${ }^{12}$. A seguir, outros autores também avaliaram as medidas do comprimento da cérvice com tecnologia 3D, demonstrando as vantagens da USG 3D em relação à $2 \mathrm{D}$ no estudo da morfologia cervical ${ }^{13}$ e a existência de diferenças entre as medidas do comprimento do colo aferidas por exame $2 \mathrm{D}$ e $3 \mathrm{D}^{14}$.

De acordo com estes estudiosos ${ }^{12,13}$, nas gestantes com variação anatômica da cérvice (colo curvo), a USG $2 \mathrm{D}$ pode sub ou superestimar a medida do verdadeiro comprimento cervical, principalmente pela dificuldade de ampla mobilização da sonda no canal vaginal, não sendo possível a identificação fiel do plano sagital do colo.

Os objetivos deste estudo foram: comparar as medidas do comprimento cervical e do afunilamento, durante a gestação, obtidas por meio da USG transvaginal 2D e 3D.

\section{Métodos}

Trata-se de estudo descritivo, prospectivo, com comparação de grupos realizado no período entre abril de 2004 e fevereiro de 2005. Este trabalho foi aprovado pelo Comitê de Ética em Pesquisa da Universidade Federal de São Paulo, e anteriormente à realização dos exames, sua metodologia foi explicada a todas as pacientes a fim de que autorizassem sua execução mediante assinatura do termo de consentimento.

Adotamos como critérios de inclusão na pesquisa as gestantes com feto vivo e período gestacional entre 19 a 24 semanas, datadas por USG de primeiro trimestre. Foram excluídas as pacientes com amniorrexe prematura, sangramento vaginal, malformação fetal e placenta prévia. A pesquisadora questionou e apurou estes dados anteriormente à realização dos exames. Foram examinadas 75 grávidas e uma paciente foi excluída da amostra por apresentar placenta prévia, restando então 74 gestantes compondo nossa casuística.

Das 74 gestantes incluídas, 58\% tinham curso superior, $56,4 \%$ eram nulíparas e $88 \%$ casadas. A idade gestacional média no momento da realização dos exames foi de $21,5 \pm 1,7$ semanas e a idade das pacientes, 29,0 $\pm 4,6$ anos

A USG cervical foi efetuada uma única vez na mesma paciente e por um único observador. As aferições das medidas do colo, no método $2 \mathrm{D}$, foram efetuadas no momento da realização dos exames, 
enquanto, no modo 3D, a biometria da cérvice foi aferida com intervalo mínimo de 7 e máximo de 15 dias da USG 2D, evitando-se dessa forma influência dos valores encontrados no exame ecográfico $2 \mathrm{D}$ e garantindo a fidelidade das medidas. Todos os exames 2D foram fotografados, e os 3D gravados em CD.

Primeiramente foi efetuada a USG cervical transvaginal 2D, após esvaziamento vesical, com a paciente em decúbito dorsal e pernas flexionadas. O equipamento utilizado foi o Voluson 730D (Kretztechnik, Áustria), o qual possui ambos os módulos, 2D e 3D com transdutor vaginal multifreqüencial 5,0-9,0 MHz.

O transdutor foi introduzido delicadamente na vagina em direção ao lábio anterior do colo. A seguir, a sonda era recuada até o ponto de perda da nitidez da imagem e reintroduzida em direção ao lábio anterior do colo, a fim de obter visibilidade total do orificio interno, externo e do canal endocervical. O canal endocervical foi identificado como linha hipoecogênica, rodeado pela área das glândulas endocervicais, estendendo-se do orifício interno ao externo.

A aferição do comprimento cervical foi efetuada no corte sagital após ampliação da imagem, ocupando em média $75 \%$ da tela do monitor, e mediante locação de calipers eletrônicos (marcadores), traçando uma reta pontilhada entre o orificio cervical externo e o interno.

$\mathrm{Na}$ presença de canal endocervical curvilineo (colo uterino curvo), a biometria foi efetuada de forma combinada (soma de duas retas). O exame durou no mínimo 5 min e duas medições foram efetuadas. A medida selecionada foi a de menor valor.

Neste estudo, foi caracterizado afunilamento a medida da dilatação e comprimento do orificio interno cervical maior ou igual a $5 \mathrm{~mm}$. Sua ocorrência foi documentada, e o mesmo foi mensurado em diâmetro (largura) e comprimento no plano sagital no modo 2D.

A seguir à realização da USG transvaginal 2D do colo uterino, utilizando-se a mesma sonda endovaginal, foi acionado o modo 3D do equipamento de ultra-som (US) para aquisição dos dados e construção da imagem 3D.

A imagem do colo exposta na tela do monitor no plano sagital foi inserida no interior de um box (dispositivo eletrônico geométrico que seleciona a estrutura a ser examinada), e a captura dos dados realizada por meio de varredura mecânica automática. As informações adquiridas foram armazenadas, processadas e, a seguir, estas foram expostas na tela do aparelho de US em organização multiplanar (MP), que possibilita a visualização da cérvice nos três cortes distintos que podem ser obtidos simultaneamente com a tecnologia 3D: sagital, axial e coronal.
A biometria do colo foi realizada por USG 3D com a imagem exposta no monitor em organização MP, e após manipulação digital, nos planos sagital e coronal, empregando técnica semelhante à aplicada no exame 2D. Vale mencionar que a exibição do corte coronal é exclusiva da tecnologia 3D. O afunilamento, quando detectado, também foi documentado e mensurado tanto em comprimento quanto em diâmetro, no plano sagital e coronal em exposição MP.

Posteriormente à aquisição de todas as medidas, os dados foram transcritos para o programa estatístico SPSS (Statistical Package for Social Sciences), versão 8.0, para Windows. O nivel de significância para todos os testes foi estabelecido em $\mathrm{p} \leq 0,05$.

Para testar diferença estatística entre os resultados médios das medidas do comprimento cervical obtidas nos exames 2D e 3D, foi utilizado o teste $t$ pareado. Também foi calculado para essas medidas o coeficiente de correlação de Pearson, objetivando-se determinar associação linear entre elas.

Em relação ao afunilamento, os dados foram categorizados e os resultados foram analisados mediante o coeficiente de concordância kappa. Para comparação das médias das medidas do diâmetro e comprimento do afunilamento nos exames $2 \mathrm{D}$ e $3 \mathrm{D}$, foi aplicado o teste $t$ pareado.

\section{Resultados}

Testando o coeficiente de correlação de Pearson, entre as medidas do comprimento cervical aferidas por USG 2D e 3D no plano sagital $(r=0,92$; $\mathrm{p}<0,001)$, USG 2D no plano sagital com 3D no coronal $(r=0,89 ; p<0,001)$ e na USG 3D entre os planos sagital e coronal $(\mathrm{r}=0,91 ; \mathrm{p}<0,001)$, detectamos correlação significante entre os métodos, demonstrando associação linear entre as medidas.

O teste $t$ pareado foi calculado para comparação das médias entre os grupos considerados. Avaliando as diferenças entre as médias das medidas do comprimento da cérvice, encontramos diferença significante entre as medidas aferidas no plano sagital por meio da USG 2D e plano coronal da USG $3 D(p=0,009)$, e na USG 3D, entre os cortes sagital e coronal $(p=0,001)$, sendo as médias das medidas do comprimento cervical ( \pm desvio padrão) de 3,9 \pm $0,92 \mathrm{c} ; 4,1 \pm 0,97$ e $3,9 \pm 0,88 \mathrm{~cm}$, respectivamente (Tabelas 1 e 2). As maiores medidas ( \pm desvio padrão) do comprimento da cérvice foram obtidas por meio da USG 3D no corte coronal $(4,1 \pm 0,97 \mathrm{~cm})$.

Comparando-se as médias das medidas do comprimento do colo, aferidas no plano sagital utilizando USG 2D e 3D, não detectamos diferença significativa $(p=0,23)$ entre elas. 
Tabela 1 - Comparação das médias das medidas do comprimento cervical aferidas por USG 2D no plano sagital e 3D no coronal.

\begin{tabular}{lccccc}
\hline Comparações & Média & $\mathbf{n}$ & DP & $\begin{array}{r}\text { Teste } t \\
\text { pareado }\end{array}$ & $\mathbf{p}$ \\
\hline $\begin{array}{l}\text { Comprimento do colo no } \\
\text { plano sagital, cm (USG 2D) }\end{array}$ & 3,9 & 74 & 0,92 & & \\
$\begin{array}{l}\text { Comprimento do colo no } \\
\text { plano coronal, cm (USG 3D) }\end{array}$ & 4,1 & 74 & 0,97 & $-2,677$ & ${ }^{*} 0,009$ \\
\hline
\end{tabular}

Teste t pareado, $p<0,05$. DP = desvio padrão.

Tabela 2 - Comparação das médias das medidas do comprimento cervical aferidas por USG 3D nos planos sagital e coronal.

\begin{tabular}{lccccc}
\hline Comparações & Média & $\mathbf{n}$ & DP & $\begin{array}{r}\text { Teste } \boldsymbol{t} \\
\text { pareado }\end{array}$ & $\mathbf{p}$ \\
\hline $\begin{array}{l}\text { Comprimento do colo no } \\
\text { plano sagital cm (USG 3D) }\end{array}$ & 3,9 & 74 & 0,88 & & \\
$\begin{array}{l}\text { Comprimento do colo no } \\
\text { plano coronal cm (USG 3D) }\end{array}$ & 4,1 & 74 & 0,97 & $-4,19$ & $0,001^{*}$ \\
\hline
\end{tabular}

Teste $t$ pareado, $p<0,05$. DP = desvio padrão.

Tabela 3 - Comparação da USG 2D com USG 3D na detecção do funil cervical.

\begin{tabular}{|c|c|c|c|c|}
\hline \multirow{2}{*}{ Afunilamento (USG 2D) } & \multicolumn{2}{|c|}{$\begin{array}{c}\text { Afunilamento } \\
\text { (USG 3D) }\end{array}$} & \multirow{2}{*}{ Kappa } & \multirow{2}{*}{$p$} \\
\hline & Sim & Não & & \\
\hline $\operatorname{Sim}$ & 7 & 1 & & \\
\hline Não & 1 & 65 & 0,860 & $<0,001^{*}$ \\
\hline
\end{tabular}

Teste kappa, $p<0,05$.

O número de afunilamento em nossa casuística foi de 9/74 exames (12,1\%). A USG 2D e $3 \mathrm{D}$ visualizaram mesmo número de afunilamento (8/9). Na USG 3D, o corte coronal detectou número maior de funil cervical que o corte sagital (8/9).

Testando o coeficiente de concordância kappa, obtivemos valor significante (kappa $=0,86$; $\mathrm{p}<0,001)$, demonstrando concordância entre os métodos 2D e 3D no diagnóstico do funil cervical (Tabela 3). Entretanto, na comparação das médias das medidas do diâmetro e comprimento do afunilamento (teste $t$ pareado), obtidas nos respectivos planos testados pelos métodos $2 \mathrm{D}$ e $3 \mathrm{D}$, não encontramos valor estatístico significativo.

As maiores medidas ( \pm desvio padrão) do diâmetro do afunilamento foram detectadas por USG $3 \mathrm{D}$ no corte coronal $(0,9 \pm 0,53 \mathrm{~cm})$, e do comprimento, na USG $2 \mathrm{D}$ corte sagital $(0,9 \pm 0,37 \mathrm{~cm})$.

\section{Discussão}

Introduzida na última década, a USG 3D tem atingido êxito nas áreas de ginecologia e obstetrícia, com destaque especial no diagnóstico pré-natal. Desde então, pesquisas científicas comparam a tecnologia 3D com a 2D nas diversas áreas da medicina, testando a sensibilidade de ambos os métodos ${ }^{15-17}$.

Existem poucos trabalhos na literatura relatando a utilização da USG 3D no estudo do colo uterino, em especial durante a gestação. De acordo com a revisão dos artigos indexados no Medline até março de 2006, oito pesquisas foram encontradas ${ }^{11-14,18-21}$. Quatro ${ }^{11,19-21}$ delas relataram a aferição do volume da cérvice em gravidez única ou múltipla; quatro ${ }^{12-14,18}$ focaram a medida do comprimento cervical.

Alguns pesquisadores ${ }^{12}$, analisando unicamente gestantes de alto risco de trabalho de parto prematuro (TPP), entre a $11^{\mathrm{a}}$ e a $32^{\mathrm{a}}$ semana, mostraram diferença significativa entre as medidas do diâmetro do funil cervical aferidas por USG 2D no plano sagital e 3D no corte coronal, e na USG 3D nos planos sagital e coronal, não evidenciando diferenças entre as medidas do comprimento da cérvice obtidas pelos métodos $2 \mathrm{D}$ e $3 \mathrm{D}$. No entanto, outros estudiosos ${ }^{13,14}$, comparando a USG 2D com o 3D para análise da biometria cervical, em grávidas da população geral, entre o segundo e terceiro trimestre de gestação, detectaram diferença significante.

Um grupo de pesquisadores da Califórnia ${ }^{14}$ evidenciou diferença significativamente maior entre as medidas do comprimento cervical aferidas por USG 2D e 3D no plano sagital. Segundo eles, quanto maior a medida do colo uterino pela USG 3D, maior a diferença entre os dois métodos de exames. É importante mencionar que estes estudiosos ${ }^{14}$ examinaram gestantes de alto ou baixo risco para parto pré-termo, no total de 34 mulheres, entre a $15^{\mathrm{a}}$ e a $36^{\mathrm{a}}$ semana, utilizando dois diferentes equipamentos de US para aquisição das medidas.

$\mathrm{Na}$ nossa opinião, a utilização de distintos equipamentos de US e transdutores com sistema 2D e 3D separados reflete na qualidade da imagem, interferindo na medição cervical, dificultando a comparação entre as técnicas. Vale ressaltar que em nosso estudo, semelhante ao de outros auto$\mathrm{res}^{18}$, os métodos 2D e 3D demonstraram boa correlação na aquisição das medidas do comprimento cervical. Na comparação das médias das medidas do comprimento do colo, foi detectada diferença estatística testando o plano coronal do exame 3D, não evidenciando diferenças no plano sagital entre os métodos 2D e 3D. 
$\mathrm{Na}$ nossa casuística, a média das medidas do comprimento cervical, mensuradas por USG $3 \mathrm{D}(4,1 \pm 0,97 \mathrm{~cm})$, foi superior a $2 \mathrm{D}(3,9 \pm 0,92 \mathrm{~cm})$, confirmando outros resultados descritos na literatura ${ }^{12-14,18}$.

Um dado importante desta pesquisa foi a detecção de variação entre as medidas do comprimento do colo obtidas por meio da USG 2D e 3D. Alguns pesquisadores ${ }^{12-14}$ descreveram em seus resultados diferença maior que $5 \mathrm{~mm}$ entre as medidas do colo uterino aferidas no plano sagital na USG 2D e 3D em 27, 48,5 e $61 \%$ dos exames realizados, respectivamente. Em nossa amostra esta variação foi detectada em 14,9\% dos casos.

Em relação ao afunilamento, a incidência em nossa casuística foi de $12,1 \%$, semelhante à de outros autores ${ }^{13}(12,6 \%)$, entretanto, divergindo dos resultados publicados por Bega et al. ${ }^{12}(56,7 \%)$.

Acreditamos que a grande incidência de afunilamento e diferenças significativas entre as médias das medidas do diâmetro presentes no trabalho de Bega et al. ${ }^{12}$ se relacionem diretamente com o perfil das pacientes examinadas (alto risco de TPP), uma vez que nosso estudo e o grupo de Severi et al. ${ }^{13}$ avaliaram gestantes da população geral e detectaram a mesma percentagem de dilatação do orifício interno do colo, não evidenciando diferença estatística entre as medidas do funil nos distintos métodos.

A USG 3D do colo uterino, na aferição das medidas no modo multiplanar, permite a manipulação digital das imagens e sua correção, favorecendo a identificação total do canal endocervical, facilitando a biometria ${ }^{12-14}$.

$\mathrm{O}$ advento da terceira dimensão propiciou medida precisa do comprimento e visualização mais detalhada da morfologia cervical. Dessa forma acreditamos que o exame USG 2D da cérvice pode ser complementado com o 3D, pois a medição errônea do colo uterino pode interferir no prognóstico da gestação, visto que atualmente a USG cervical é exame de relevância no rastreamento do TPP, principalmente em gestantes de alto risco de parto pré-termo ${ }^{22}$.

Diante das diferenças evidenciadas em nossos resultados, na aferição da biometria cervical utilizando a USG 2D e 3D, acreditamos que a USG 3D pode acrescentar-se aos exames da cérvice atualmente empregados no rastreamento do trabalho de parto prematuro; no entanto, o exame 3D do colo encontra-se em fase experimental, sendo necessários, novas publicações para a confirmação desses resultados para implantação do método na prática clínica.

Na nossa opinião, o entusiasmo do emprego da USG 3D em obstetrícia atribui-se à maior precisão diagnóstica das malformações fetais ${ }^{23}$ e à perspectiva de aumentar o valor preditivo do exame ultra-sonográfico cervical, mediante pesquisa de novos parâmetros biométricos e marcadores morfológicos do colo, que aumentem a sensibilidade do método, visando a prevenção do trabalho de parto pré-termo.

\section{Referências}

1. Sarti DA, Sample WF, Hobel CJ, Staisch KJ. Ultrasonic visualization of a dilated cervix during pregnancy. Radiology. 1979;130(2):417-20.

2. Varma TR, Patel RH, Pillai U. Ultrasonic assessment of cervix in normal pregnancy. Acta Obstet Gynecol Scand. 1986;65(3):229-33.

3. Lewin D, Sadoul G, Sylvain-Leroy BS. Perineal echotomography. A new method of objective measurement of the cervix. J Gynecol Obstet Biol Reprod (Paris). 1976;5(5):651-5.

4. Brown JE, Thieme GA, Shah DM, Fleischer A, Boehm FH. Transabdominal and transvaginal endosonography: evaluation of the cervix and lower uterine segment in pregnancy. Am J Obstet Gynecol. 1986;155(4):721-6.

5. To MS, Skentou C, Cicero S, Nicolaides KH. Cervical assessment at the routine 23-week's scan: problems with transabdominal sonography. Ultrasound Obstet Gynecol. 2000;15(4):292-6.

6. Ayers JW, DeGrood RM, Compton AA, Barclay M, Ansbacher R. Sonographic evaluation of cervical length in pregnancy: diagnosis and management of preterm cervical effacement in patients at risk for premature delivery. Obstet Gynecol. 1988;71 (6 Pt 1):939-44.

7. Andersen HF, Nugent CE, Wanty SD, Hayashi RH. Prediction of risk for preterm delivery by ultrasonographic measurement of cervical length. Am J Obstet Gynecol. 1990;163(3):859-67.

8. Iams JD, Paraskos J, Landon MB, Teteris JN, Johnson FF. Cervical sonography in preterm labor. Obstet Gynecol. 1994;84(1):40-6.

9. Iams JD, Goldenberg RL, Meis PJ, Mercer BM, Moawad A, Das A, et al. The length of the cervix and the risk of spontaneous premature delivery. National Institute of Child Health and Human Development Maternal Fetal Medicine Unit Network. N Engl J Med. 1996;334(9):567-72.

10. Sonek J, Shellhaas C. Cervical sonography: a review. Ultrasound Obstet Gynecol. 1998;11(1):71-8.

11.Hoesli IM, Surbek DV, Tercanli S, Holzgreve W. Three dimensional volume measurement of the cervix during pregnancy compared to conventional 2D-sonography. Int J Gynaecol Obstet. 1999;64(2):115-9.

12.Bega G, Lev-Toaff A, Kuhlman K, Berghella V, Parker L, Goldberg B, et al. Three-dimensional multiplanar 
transvaginal ultrasound of the cervix in pregnancy. Ultrasound Obstet Gynecol. 2000;16(4):351- 8.

13. Severi FM, Bocchi C, Florio P, Picciolini E, D`Aniello G, Petraglia F. Comparison of two-dimensional and three-dimensional ultrasound in the assessment of the cervix to predict preterm delivery. Ultrasound Med Biol. 2003;29(9):1261-5.

14. Towner D, Boe N, Lou K, Gilbert WM. Cervical length measurements in pregnancy are longer when measured with three-dimensional transvaginal ultrasound. J Matern Fetal Neonatal Med. 2004;16(3):167-70.

15. Maymon R, Herman A, Ariely S, Dreazen E, Buckovsky I, Weinraub $Z$. Three-dimensional vaginal sonography in obstetrics and gynaecology. Hum Reprod Update. 2000;6(5):475-84.

16. Cho KR, Seo BK, Lee JY, Pisano ED, Je BK, Lee JY, et al. A comparative study of 2D and 3D ultrasono-graphy for evaluation of solid breast masses. Eur J Radiol. 2005;54(3):365-70.

17. Moeglin D, Talmant C, Duyme M, Lopez AC; CFEF. Fetal lung volumetry using two- and three-dimensional ultrasound. Ultrasound Obstet Gynecol. 2005;25(2): 119-27.

18. Rovas L, Sladkevicius P, Strobel E, Valentin L. Reference data representative of normal findings at two-dimensional and three-dimensional gray-scale ultrasound examination of the cervix from 17 to 41 weeks' gestation. Ultrasound Obstet Gynecol. 2005;27(4):392-402.

19.Strauss A, Heer IM, Fuchshuber S, Janssen U, Hillemanns P, Hepp H. Sonographic cervical volumetry in higher order multiple gestation. Fetal Diagn Ther. 2001;16(6):346-53.

20.Strauss A, Muller-Egloff S, Heer IM, Dannecker C, Hepp H. [Cervical incompetence in multifetal gestation: diagnosis and prophylaxis]. Gynakol Geburtshilfliche Rundsch. 2003;43(2):91-7. German.

21.Rozenberg P, Rafii A, Senat MV, Dujardin A, Rapon $\mathrm{J}$, Ville Y. Predictive value of two-dimensional and three-dimensional multiplanar ultrasound evaluation of the cervix in preterm labor. J Matern Fetal Neonatal Med. 2003;13(4):237-41.

22.Hoesli I, Tercanli S, Holzgreve W. Cervical length assessment by ultrasound as a predictor of preterm labor - is there a role for routine screening? BJOG. 2003;110 Suppl 20:61-5.

23. Merz E, Bahlmann F, Weber G. Volume scanning in the evaluation of fetal malformations: a new dimension in prenatal diagnosis. Ultrasound Obstet Gynecol. 1995;5(4):222-7. 